FACTA UNIVERSITATIS

Series: Automatic Control and Robotics Vol. 17, $\mathrm{N}^{\mathrm{o}}$ 2, 2018, pp. 105 - 116

https://doi.org/10.22190/FUACR1802105M

\title{
INTRODUCTION TO NANOTECHNOLOGY AND MOLECULAR SELF-ASSEMBLY*
}

\author{
$U D C((620.3: 66.017): 539.2)$
}

\section{Jelena Manojlović}

University of Niš, Faculty of Mechanical Engineering, Republic of Serbia

\begin{abstract}
What is nanoscience? What is nanotechnology? What is so special about the nanoscale? These questions are only a few important ones to discuss in order to understand the nanoworld. Nanoscience and nanotechnology were described to introduce the basic ideas of the new technology that can significantly change our life. The nanoworld is invisible to the naked eye, and with many unusual properties of material. It has been observed at the nanoscale that numerous properties, such as the melting point, electrical conductivity, or chemical reactivity, change as a function of the size of the sample, and (this is true for) many nanomaterials that have been produced. Special attention in this paper is focused on the formation of self-assembled monolayers. This process is described as the creation of organic thin films of nanometer thickness, and it is an emerging area of materials chemistry, utilized in many applications. In order to facilitate the understanding of self-assembled films formation, the paper describes the adsorption of quaternary ammonium surfactants onto relatively simple inorganic substrates such as mica.
\end{abstract}

Key words: nanotechnology, nanoscience, nanomaterials, boundary lubrication, selfassemble monolayers

\section{NANOTECHNOLOGY AND NANOSCIENCE}

Nanotechnology is a new science, the study of the phenomena at the scale of atoms and molecules. When talking about the nanometer scale we mean the size between 1 and 100nm. A nanometer is, for example, a million times smaller than the smallest measurement on a

Received March 26, 2018

Corresponding author: Jelena Manojlović

Faculty of Mechanical Engineering, Aleksandra Medvedeva 14, 18000 Niš, Republic of Serbia

E-mail: jelena.manojlovic@masfak.ni.ac.rs

* Acknowledgement: The author would like to gratefully acknowledge the support in obtaining and understanding the presented results provided by her mentors, Nicholas D. Spencer and Manfred Heuberger, members of the Laboratory for Surface Science and Technology (LSST), a part of the Department of Materials at the ETH Zurich, Switzerland, where all the experiments were performed. 
ruler; every second a human fingernail grows 1 nanometer; or 10 hydrogen atoms lined up measure about $1 \mathrm{~nm}$. Bearing in mind the possibility to measure, manipulate and organize matter at this scale, there is a big change occurring in science and technology. As a result of the research at the nanoscale, many new organized structures of matter have been made possible, such as carbon nanotubes, molecular motors, nanorobots and many others $[1,2]$.

In addition to studying the phenomena that exist at the nanolevel, nanotechnology includes the manufacturing technology described as the molecular manufacturing, where researchers try to design and produce very small machines, nano-machines, by building objects atom by atom [2,3]. Even at the middle of the last century some scientists visualized very small computers and machines made of atoms, which was very advanced at that time [4].

Nano is a very popular area of science and technology today, with significant upgrades and great attention paid by researchers. The reason for the growing progress and popularity of this area lies in numerous important inventions [1]. Nanoscience is an interdisciplinary science, and its studies involve many other sciences, such as chemistry, physics, engineering, material science and computer science [5]. Of course, there are still many open questions and topics interesting and important to researchers at such a small scale, which can be helpful for the development of science in general.

There are many definitions of nanoscience and nanotechnology in the literature [4] and more generalized ones are given here. Nanoscience can be defined as the study of objects and phenomena occurring at the scale of 1 to $100 \mathrm{~nm}$ and the manipulation of materials at this scale. Nanotechnology can be defined as the understanding and control of matter at dimensions of roughly 1 to 100 nanometers, using the knowledge of nanoscience for applications. The difference between nanotechnology and nanoscience can be described in the same manner as the difference between technology and science in general [6].

Due to the fast and significant development of nanotechnology and nanoscience, new nanometerials have been produced and applied in the appropriate fields. The unusual properties of carbon nanotubes make them applicable in electronic devices or chemical sensors [7]. The existence of self-cleaning windows or certain surfaces is the consequence of the nanotechnology research [8]. Numerous cosmetic products have been made on the basis of the knowledge in nanotechnology [5, 9].

An emerging field in nanotechnology is nanorobotics, and due to very small, atomic and molecular-sized objects, is called molecular robotics. The task of this technology is to create, produce and apply very small robots at the nanometer scale. A nanorobot is a specialized nanomachine with the dimensions of typically 0.5 to 3 microns with $1-100 \mathrm{~nm}$ parts, which requires very little energy to operate. The production of nanorobots is the subject of an enormous interest in many technology fields today [10, 11]. Special attention is focused on the application of nanorobotics in medicine ranging from neurosurgery to dentistry [12].

\section{HISTORY OF NANOTECHNOLOGY}

The term nanotechnology is usually connected to Mr. Richard Feynman. In his lecture from 1959, the possibility to create products of nanoscale dimensions by atoms or molecules was considered for the first time. Feynman says: "The principles of physics, as far as I can see, do not speak against the possibility of maneuvering things atom by atom" [4]. 
The word "nanotechnology" was first introduced in 1974. At the international conference in Tokyo, the Japanese scientist Norio Taniguchi used this word to describe very small mechanisms of the nanometer size. Therefore, nanotechnology is a young scientific field, with many opportunities for further development [2].

There are several nano visionaries known in the scientific literature, especially important for the beginning of the development of this scientific field. One of them is Eric Drexler who introduced the concept of development of very small nanorobots with the ability to repair medical problems inside the human body [11]. The idea of nanotechnology created by R. Feynman was further improved by E. Drexler and described in his book "Vehicles of creation: the arrival of the nanotechnology era" published in 1986.

In the modern history of nanotechnology, in addition to the above-mentioned work, there are few important years: 1981 - the invention of the scanning tunneling microscope (STM) by G. Binning and H. Rohrer, 1985 - the discovery of fullerens, 1986 - the first atomic force microscope (AFM). With the new invented equipment, such as STM and AFM, it became possible to view surfaces at the atomic scale and in the late 1990s and at the beginning of this century almost all scientific fields were influenced by nanotechnology [6].

\section{EXPERIMENTAL TECHNIQUES IN NANOTECHNOLOGY}

A very important field of study in nanotechnology today is the investigation of nanostructures, the behavior research of materials and processes at the atomic and molecular levels. The reason for that is the complexity of the research at the nanoscale and the equipment required for the measurements at the nanoscale - what we want to see determines which instruments we use [6]. Indeed, besides the theoretical knowledge and findings, there is a need for new equipment and new tools, to see structures at the nanoscale and to change them.

The naked eye can see down to about 20 micrometers. With the light microscopes one can see down to about 1 micrometer, which indicates that these cannot be used to detect small particles on the nanoscopic scale. Therefore, the electron microscopes have been developed to overcome the limitations of light microscopes, limited by the physics of light. For that purpose, new characterization methods and techniques have been created during the last forty years [5]. A few of them are shortly described here.

A scanning electron microscope (SEM) is a type of electron microscope that images a sample by scanning it with a high-energy beam of electrons. The electrons interact with the atoms of the sample surface and produce signals. These signals contain information about the sample, such as surface topography, composition, or electrical conductivity. SEMs can achieve resolution better than 1 nanometer [5].

A family of tools used to form images of surface at the nanoscale is defined as scanning probe microscopes (SPMs). A specific characteristic of these microscopes is that they have a physical probe to scan the surface of a researched sample and generate an image of the surface. In addition to visualizing nanoscale structures, there are certain SPMs with the ability to manipulate individual atoms and move them on the substrate [5].

There are several types of SPMs, such as the Atomic Force Microscopes (AFMs) that measure the electrostatic forces between the cantilever tip and the sample. Another 
instrument, called the Scanning Tunneling Microscope (STM), can measure the electrical current flowing between the cantilever tip and the sample $[5,13]$.

\section{NANOMATERIALS}

There is a big difference between properties of bulk materials and nanostructures. Many characteristics of materials, physical, chemical and biological, vary significantly at the level of nanometer. For example, bulk gold looks yellow in color, and nano sized gold appears red in color [14]. A certain number of atoms or molecules bound together with a radius of $100 \mathrm{~nm}$ or less may have very different properties as compared to the same atoms bonded in bulk materials. Therefore, one can conclude that nanosized particles exhibit properties that differ from those of larger particles of the same substance [15]. Hence, the study of the phenomena at the nanoscale can help us to learn more about the nature of matter, discover new phenomena, and open many questions about the behavior of molecules on the nanometer scale. A special advantage of these results and studies is the possibility to figure out the procedure of making new materials and products that have a great influence on technology and our life [2].

A nanoscale material can be defined as a substance where at least one dimension is less than 100 nanometers. These materials are important because at the nanometer scale they have unique electrical, magnetic or optical properties. Due to their specific properties, nanomaterials have a big impact on their application in numerous technical fields, medicine and medicaments, or in the commercial products such as cosmetics and sporting goods $[5,9]$.

One very interesting and today very well-known example of nanomaterials is a carbon nanotube. This material is an allotrope of carbon with a cylindrical nanostructure and it is 100 times stronger than steel. Nanotubes are very often applied in new nanoproducts [2].

As described above, the properties of materials with nanometer dimensions are significantly different from those of bulk materials. There are big length scale differences between the macroscopic scale and nanoscale, and the different forces dominate (gravitational forces become negligible and electromagnetic forces dominate). Usually, scientists suggest several important reasons why nanoscale materials may differ from macro-scale materials. Instead of the classical mechanics, the laws of quantum physics are used to describe energy and motion and behavior in the nanoworld. Due to a smaller size of nanoparticles, a greater amount of the substance comes into the contact with surrounding molecules, and the nanosize material becomes more reactive with the molecules around it [15].

The question that arises is: how is it possible to build things that are so small? There are many methods that can be used to produce nanomaterials, such as the two approaches defined in nanotechnology, called "top-down" and "bottom-up". The top-down approach is defined as the process of the creation of small devices by consecutive cutting of a bulk material to get nanosized objects. The bottom-up approach implies the arrangement of small components and formation of more complex assemblies [5].

One of the fabrication methods, as described above, is the atom-by-atom assembly, or moving atoms from one place to another by the AFM and STM. Another procedure capable of creating new structures is the self-assembly process. This process already 
exists in nature (e.g. cell membranes), and it is usually a very simple technique, where the components from a disordered system assemble, organize themselves, and form units of appropriate properties such as ultrathin films of controlled thickness. Because of this, the process of self-assembly will be described in more details.

\section{THE PROCESS OF SELF-ASSEMBLY}

An important aspect of bottom-up approaches is the molecular self-assembly. This area of nanoresearch is very wide and important because of the ability of morphological modification (coating) of a surface by the process of self-assembly [13]. It was noticed that a large number of organic compounds has the ability to form organic thin films on solid surfaces. Such films can exhibit special optical, mechanical, chemical or electrical properties, which is frequently needed, and can be applied in many areas such as electronic components [16] or in biomedical applications $[17,18]$. One of the most elegant ways to make ultrathin organic films of controlled thickness is to prepare self-assembled monolayers (SAMs), considered as a very important example of equilibrium structural organization on the molecular scale (nanoscale) [19, 20].

Interest in SAMs formation and structure has been increasing recently, because selfassembly is a promising approach for the generation of atomically structured devices. There is also a special interest in the possibility of manufacturing molecular layers of particular properties. The molecular self-assembly is recognized as a powerful strategy for the fabrication of nanoscale structures [21]. By developing a number of powerful techniques in surface analysis, academic interest in SAMs has regained its importance because of the possibilities to investigate the growth, stability, film thickness or molecular order in such a thin film $[22,23]$.

SAMs have many applications in controlling surface properties in microelectromechanical devices [24], controlling and limiting surface corrosion [25], producing nanoscale organic transistors [26], or in biology to immobilize biological molecules onto monolayers as a platform for biosensor applications [27].

\subsection{Surfactants self-assembly}

Self-assembled monolayers (SAMs) are formed spontaneously when certain classes of molecules adsorb onto a solid surface from a solution. By this procedure, a very thin layer defined as a self-assembled monolayer is formed on the substrate. SAMs can be prepared using different substrates and different types of molecules able to adsorb on the substrate. The choice of the substrates used in the self-assembling process depends on the molecules and their interactions, as well as the final application of the surfaces covered by SAMs.

Surfactants are organic compounds very often applied in SAMs formation. These molecules are amphiphilic, which means that they have two parts, the hydrophilic head group and the hydrophobic tail. The typical structure of the organic thin film formed by surfactants is illustrated in Fig. 1. 


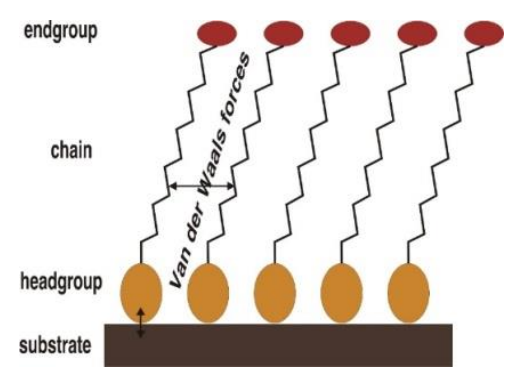

Fig. 1 An organized monolayer on a substrate

The hydrophilic head group of the surfactant molecule usually has an attractive interaction with the substrate surface. The hydrophobic tail of the molecule without a strong interaction with the surface can be chemically tailored to exhibit specific chemical properties. Therefore, SAMs offer the capability to form ordered organic surface coatings, suitable for various applications such as wetting or corrosion protection [28].

In the process of self-assembly two important forces can be distinguished : the bonding of surfactant molecules to the substrate, and the interactions between molecules in the monolayer (Fig. 1), where the balance between these two forces can be shifted according to the properties of the substrate and the surfactant. The monolayer can be stable or metastable, and the stability of the formed layer is mainly determined by the strong inter-chain van der Waals interactions [29], and therefore a variety of surface structures, such as monolayer, bilayer or multilayer can form on the substrate [30].

The ability of surfactant molecules to adsorb at the solid-liquid interface depends on the properties of all the components of the system such as the solid, the surfactant, and the solvent used to prepare surfactant solution [31]. In addition to the above factors, there are many other factors that influence the thin film formation, such as $\mathrm{pH}$, the concentration of surfactant molecules, or even environmental conditions such as ambient temperature and humidity [32]. Bearing in mind that the thickness of the formed SAMs can be in the order of nanometer, it is not surprising that a large number of parameters influence its properties.

\subsection{Adsorption of cationic surfactants}

Due to the flexibility in choosing the molecular architecture, organic molecules have many interesting applications, such as biosensors, in nonlinear optics, for lubrication or in controlling water adsorption. Therefore, in recent years, much attention has been directed to the study of SAMs. However, a discrepancy still exists between the theoretical understanding and the practical importance involved in the formation of such layers.

Cationic surfactants are a small subgroup of surfactants with some unique properties that are not present in other surfactants. Many authors have aimed at investigating the adsorption of cationic surfactants from aqueous solutions onto a variety of solid surfaces including graphite [33], silica and mica [34]. This process has been widely studied usually by different techniques such as x-ray photoelectron spectroscopy (XPS), the surface forces apparatus (SFA) or contact angle (CA) measurements [35].

Preparing self-assembled monolayers is one of the most elegant ways to make ultrathin organic films of controlled thickness, and it represents attractive model systems for boundary lubrication. Organic thin films are an emerging area of materials chemistry and 
are utilized in many areas of application. The molecular self-assembly is recognized as a powerful strategy for the fabrication of nanoscale structures [36, 37, 38].

In order to describe the adsorption process to form SAMs, our attention in the next chapter will be focused on the modification of the muscovite mica surface by adsorption of quaternary ammonium surfactants (cetyltrimethylammoniumbromide - CTAB), with the aim to produce hydrophobic and well-ordered homogeneous monolayers. Adsorbed layers were prepared at certain concentrations of an aqueous surfactants solution.

The original goal of these experiments was to produce self-assembled monolayers and use them as model systems to study boundary lubrication. But, there was a problem concerning the results being repeated, as well as the characterization of the adsorbed CTAB layers on muscovite mica in detail. The various SAM morphologies demonstrate the influence of a large number of experimental parameters on the adsorption process, especially temperature and humidity, which is rarely described in the literature.

\subsection{CTAB adsorption at muscovite mica as an example of SAMs formation}

The adsorption of quaternary ammonium surfactants onto mica has frequently been studied [39]. Several aspects of the adsorption of cationic surfactants onto mica have been studied in details, but the interactions between the mica surface and the alkylammonium ions have not yet been studied closely.

Following the literature it is possible to find various proposed adsorption protocols for $\mathrm{CTAB}$ adsorption on mica, with some significant differences in the conditions under which the adsorption takes place. Depending on the conditions, it has been reported that $\mathrm{CTAB}$ adsorbs on mica as a compact monolayer, as a stable hydrophobic surface, as a bilayer or forms aggregates. For the preparation of CTAB self-assembled films on mica, numerous adsorption protocols have been proposed in the literature. The applied procedures include the variation of many parameters, such as different temperatures in SAM preparation, the adsorption time or the post-adsorption sample treatment. Different conclusions about adsorption theories and the existence of numerous mechanisms underline this broadness $[40,41]$.

Adsorbed layers were prepared on mica using certain concentrations of an aqueous surfactants solution following our own designed protocol. To characterize and determine the properties of SAMs, two techniques were extensively applied in this work, contact angle measurements and the atomic force microscopy (AFM).

Experimental procedure. In our experiments we used a quaternary ammonium surfactant, single-tailed cetyltrimethylammonium bromide, (CTAB), with the molecular structure $\mathrm{CH}_{3}\left(\mathrm{CH}_{2}\right)_{15} \mathrm{~N}^{+}\left(\mathrm{CH}_{3}\right)_{3} \mathrm{Br}$. To prepare the CTAB solution, as a solvent, ultrapure water with appropriate properties was used. All experimental tools (tweezers and bottles) were cleaned before the experiments to minimize any contamination. For the adsorption experiments, very small pieces of muscovite mica $\left(1-1.5 \mathrm{~cm}^{2}\right)$ were cut by scissors, and further freshly cleaved mica samples on both sides were immersed into the surfactant solution.

To simplify the procedure, it is suitable to define a few important experimental steps: adsorption, rinsing, drying and SAMs analysis (CA-contact angle measurements and AFM analysis). An adsorption protocol named "CTAB in/CTAB out", shown in Fig. 2, contains the immersion and extraction of a mica sample into and out of the surfactant solution. 


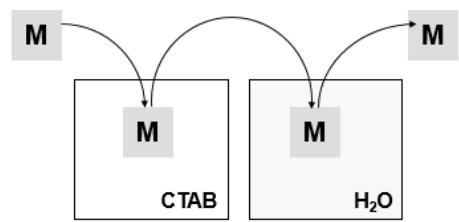

Fig. 2 "CTAB in, CTAB out" adsorption protocol

After adsorption in the CTAB solution (30 seconds), the sample was rinsed in ultrapure water (10seconds) to remove the excess solution and excess surfactant molecules. After these two steps, the mica sample was dried with a clean nitrogen stream. Then, the sample was ready for further analysis (CA and AFM).

In our adsorption experiments a $1000 \mathrm{ml}$ stock solution of $10^{-2} \mathrm{M}$ CTAB was prepared at room temperature. Due to low solubility of CTAB in water, the solution was heated to $30-35^{\circ} \mathrm{C}$. By diluting this solution, carried out by adding the appropriate amount of ultrapure water, we prepared the surfactant solution of the chosen concentrations and the adsorption results at one of the concentrations, $10^{-4} \mathrm{M}$, will be described here.

Results: Using the above-described preparation protocol, a significant number of samples were prepared. The AFM images of two representative samples obtained by the "CTAB in/CTAB out" protocol at certain concentration are shown in Fig. 3. In both groups of experiments, it has to be emphasized that temperature in the laboratory was not controlled and measured. The temperatures were assumed to be around $30^{\circ} \mathrm{C}$ and around $23^{\circ} \mathrm{C}$.

The advancing and receding water contact angles (advancing $\mathrm{CA} /$ receding $\mathrm{CA}$ respectively) have been measured in several spots on the sample covered by the CTAB surfactant. At the laboratory temperature of around $20^{\circ} \mathrm{C}$ the results of CA measurements for the representative sample were $85^{\circ} / 30^{\circ}$, and for the CTAB layer formed at around $30^{\circ} \mathrm{C}$ the detected advancing and receding $\mathrm{CA}$ on the presented sample were $82^{\circ} / 22^{\circ}$, such as shown in Figs. $3 \mathrm{a}$ and $3 \mathrm{~b}$. The advancing and receding water contact angle measured on both samples exhibited hysteresis. The similar results were recorded at numerous samples prepared at the same experimental conditions.
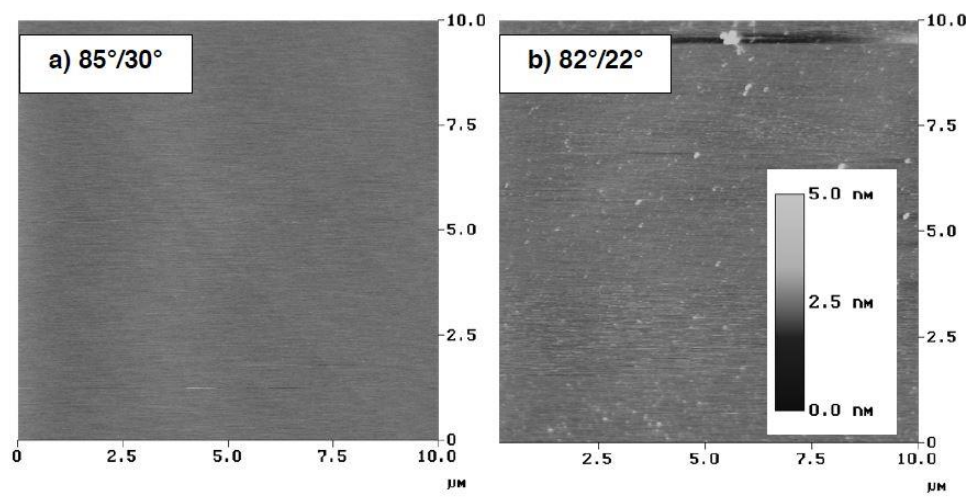

Fig. 3 AFM images of CTAB on mica obtained with the "CTAB in/CTAB out" protocol at a concentration of $10^{-4} \mathrm{M}$. Also shown are advancing and receding water contact angles at different laboratory temperatures: a) at around $20^{\circ} \mathrm{C}$ and b) at around $30^{\circ} \mathrm{C}$ 
A clear temperature influence on SAMs adsorption was observed. On the sample prepared at $30^{\circ} \mathrm{C}$ (Fig. $3 \mathrm{~b}$ ), a significant number of clusters with the height between $0.5 \mathrm{~nm}$ to $3.8 \mathrm{~nm}$ and the size around $250 \mathrm{~nm}$ size were detected, according to the gray scale of the image, which represents a height range of $5 \mathrm{~nm}$ (cf. Fig. 3.b). The sample prepared at the lower temperature, $20^{\circ} \mathrm{C}$, was more promising and homogeneous.

Discussion: The method of contact angle (CA) measurements is a quantitative measure of the thermodynamic surface energy balance between a solid, a liquid and a gas phase. Geometrically, CA is defined as the angle formed at the three-phase boundary, where the liquid, the gas and the solid meet. In SAMs analysis, the contact angle measurement is the usually selected procedure, because it is a very simple and fast process, convenient for a quick check of SAM quality.

Contact angle measurements were conducted on the representative samples to verify the hydrophobicity of SAMs that covered mica surfaces. This information is very important, because in a potential boundary lubrication regime we have planned to examine properties of two hydrophobic surfaces - mica surfaces covered by SAMs on both surfaces, at a very small distance (Fig. 4).

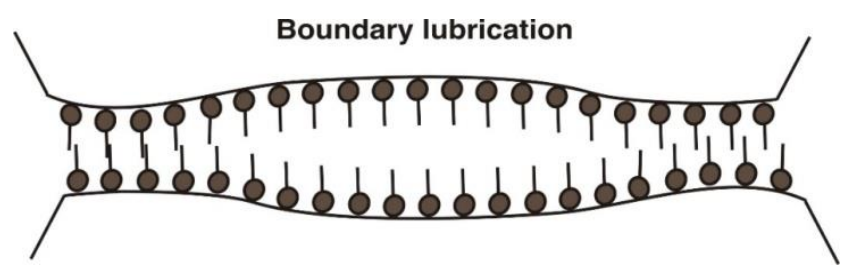

Fig. 4 Boundary lubrication

The results can be interpreted on the basis of certain rules: contact angle measurements indicate good wetting by very low $\left(<30^{\circ}\right)$ contact angles (e.g. water on hydrophilic surfaces), neutrality at a contact angle near $60^{\circ}-90^{\circ}$ and non-wetting is observed by a contact angle greater than $90^{\circ}$ (determined on hydrophobic surfaces). From the CA measurements in all groups of experiments, due to advancing contact angles between $75^{\circ}$ and $90^{\circ}$, it was concluded that hydrophobic surfaces were achieved. The aim of our experiments was to produce homogeneous monolayers of surfactants on muscovite mica by the chosen quaternary ammonium surfactant - CTAB, but the significant hysteresis in these results suggests a chemical heterogeneity or roughness in the surfactant film [42].

In order to get more information about SAMs properties, it is important to include more than one method of surface analysis. For that purpose the AFM measurements have been applied very often, and for this mica-CTAB system very inhomogeneous surfactant layers were determined, as described in the literature [13]. In our AFM results presented in Fig. 3, the various SAM morphologies were found in adsorption experiments, indicating that the temperature during solution preparation, frequently neglected, can have a crucial influence on the surfactant solution structure and the adsorption mechanism on the substrate. The variety of CA and AFM results indicates very complex molecular mechanisms in the process of surfactant self-assembling. The cause of this is the fact that many experimental parameters can change in the adsorption experiment (temperature, solution $\mathrm{pH}$, humidity, ion-exchange capacity of muscovite mica etc.). Therefore, there is 
a need to control many parameters in order to clarify the mechanisms that are happening at the nanoscale.

Another problem in adsorption experiments is the repetition of the results. The various SAM morphologies, found on mica under different adsorption conditions, demonstrate the influence of a large number of experimental parameters on the adsorption process, as previously concluded. This observation is rarely described in the literature.

The large differences in the protocols, which exist in the literature, make it difficult to predict the optimum procedure to obtain a homogeneous surfactant film on mica. Model hydrophobic surfaces, formed by surfactant adsorption on mica, have often been assumed to be stable layers. The stability and structure under the experimental conditions is often unverified. It is important to note that the aggregate structure in a solution, such as micelles or hemimicelles, according to our knowledge, has never been considered as an important factor for adsorption.

Due to that, we have organized our own adsorption experiment (Fig. 2) and analyzed samples in order to define the most advantageous protocol. The remarkable variety of film morphologies spurred us to go back even one more step and to study the properties of CTAB solutions. We have investigated the properties of aqueous CTAB solutions via specific conductivity measurements as a function of temperature [19].

These adsorption processes are described here to point out that self-assembling is a simple, yet very complex procedure, with the influence of the numerous parameters in all experimental steps needed to be considered and carefully defined. The surfactant films on mica, formed according to the described experimental protocol, were characterized by contact angle measurements and by AFM. A variety of SAMs are influenced by temperature. These results suggested that temperature can influence all steps in the adsorption procedure, from the solution preparation to the rinsing step. The fact that the CTAB solution properties changed remarkably around room temperature, makes this system appear particularly complex [13].

\section{CONCLUSIONS}

The word nanotechnology is new, as well as the technology and science connected to this. The structures and devices produced at the nanometer scale are in dimensions of the order of a billionth of a meter. Based on the findings in nanotechnology, today's scientists are able to create new materials and new devices. They have many applications in engineering, nanoelectronics, and nanomedicine, biomaterials, but also in products for our everyday life. Nanotechnology includes numerous fields of science, such as surface science, organic chemistry, biology, semiconductor physics, molecular engineering and many others.

Molecular nanotechnology, or molecular manufacturing, describes nano-systems operating on the molecular scale. As an example of molecular manufacturing, the formation of an organic thin film by the adsorption of quaternary ammonium surfactants onto the atomically smooth muscovite mica surface has been described. It was observed that the morphology and the molecular order of self-assembled monolayers depend on many experimental parameters, such as temperature, solution concentration and many others. It was shown that a high stability of the adsorbed films is very rarely detected. A 
reproducible stability of the resulting films, however, remains an issue, pointing to the complexity of producing a very well-ordered system at the nanoscale. In this model case of quaternary ammonium surfactants, the formation of homogeneous, well-ordered and reproducible monolayers is a very challenging task. In order to assess such complex systems, a systematic variation of a great number of parameters was a necessary procedure.

\section{REFERENCES}

[1] M. Kuno, Introduction to nanoscience and nanotechnology. 2014. (ISBN-10: 1505583233 )

[2] A. Nouailhat, An introduction to nanoscience and nanotechnology. Hermes Science/Lavoisier in 2006, France, (ISBN 978-1-84821-007-3).

[3] M. Rieth, Nano-Engineering in Science and Technology: An Introduction to the World of Nano-Design. World Scientific Publishing Company 2003, ( ISBN-10, 9812380736)

[4] D. C. Agrawal, Introduction to nanoscience. Oxford University Press Inc., New York, 2013 (ISBN: 978981-4397-97-1)

[5] L. Filipponi, D. Sutherland, Introduction to nanoscience and nanotechnologies. 2010. [Online]. Available: http://nanoyou.eu/attachments/188_Module-1-chapter-1.pdf

[6] M. Ratner, D. Ratner, Nanotechnology. Pearson Education, Inc., 2006. [Online]. Available: http://materialrulz.weebly.com/uploads/7/9/5/1/795167/nanotechnology_a_gentle_introduction_to_the_ next_big_idea.pdf

[7] W. Obitayo, T. Liu, "A Review: Carbon Nanotube-Based Piezoresistive Strain Sensors," Journal of Sensors, vol. 2012, doi:10.1155/2012/652438

[8] J. Manojlovic, Friction at nanoscale-self-assembled monolayers, pp. 153-175. Commercialization of nanotechnologies-A case study approach, Springer international publishing, 2018.

[9] S. Raj, S. Jose, U.S. Sumod, M. Sabitha, "Nanotechnology in cosmetics: Opportunities and challenges, " J. Pharm Bioallied Sci., vol. 4, no. 3, pp. 186-193, 2012. ( doi: 10.4103/0975-7406.99016 )

[10] B. Balan, S. Narayanan, NANO ROBOTICS - ITS TIME FOR CHANGE, International Journal of Oral Care \& Research, Jul - Sep 2014; Volume 2, Issue 5, pp.41-46

[11] N. A. Weir, D. P. Sierra, J. F. Jones, A review of research in the field of nanorobotics, Sandia report, SAND 2005-6808, 2005.

[12] B.S. Saadeh, M. D. Vyas, "Nanorobotic application in medicine: Current proposals and design," American Journal of Robotic Surgery, vol. 1, no. 1, pp. 4-11, 2014. (doi:10.1166/ajrs.2014.1010.)

[13] J. Manojlovic, Structure, morphology and history effects in surfactant self-assembly. Dissertation, ETH Zuerich (2006)

[14] G. Schmid, B. Corain, "Nanoparticulated Gold: Syntheses, Structures, Electronics, and Reactivities," European Journal of Inorganic Chemistry, vol. 2003, no. 17, pp. 3081-3098, 2003. (doi:10.1002/ ejic.200300187)

[15] J.T. Lue, Physical Properties of Nanomaterials. Encyclopedia of Nanoscience and Nanotechnology X:146, (2007)

[16] B. Kumar, K.B. Kumar, Y.S. Negi, "Organic Thin Film Transistors: Structures, Models, Materials, Fabrication, and Applications: A Review, " Polymer Reviews, vol. 54, no.1, pp. 33-111, (2014). (https://doi.org/10.1080/15583724.2013.848455)

[17] J.D Swalen, D. L. Allara, J. D. Andrade, E. A. Chandross, S. Garoff, J. Israelachvili, T. J. McCarthy, R. Murray, R. F. Pease, and et al., "Molecular Monolayers and Films," Langmuir, vol. 3, no. 6, pp. 932-950, 1987. ( doi:10.1021/la00078a011

[18] A. Singh, K. Suh, "Biomimetic patterned surfaces for controllable friction in micro- and nanoscale devices," Micro and Nano Systems Letters 1:6, 2013.( doi: 10.1186/2213-9621-1-6)

[19] J. Manojlovic, "The Krafft Temperature of Surfactant Solutions," Thermal Science, vol. 16, no. 2, pp. S631-S640, 2012. (doi: 10.2298/TSCI120427197M)

[20] G.M. Whitesides, B. Grzybowski, "Self-assembly at all scales," Science, vol. 295, no. 5564, pp. 24182421, 2002. (doi: 10.1126/science.1070821)

[21] J.M. Mellott, D.K. Schwartz, "Supercritical self-assembled monolayer growth," Journal of the American Chemical Society, vol. 126, no. 30, pp. 9369-9373, 2004. (doi:10.1021/ja0489588) 
[22] R.W. Carpick, M. Salmeron, "Scratching the surface: Fundamental investigations of tribology with atomic force microscopy," Chemical Reviews, vol. 97, no. 4, pp. 1163-1194, 1997. (doi: 10.1021/cr960068q)

[23] D.K. Schwartz, "Mechanisms and kinetics of self-assembled monolayer formation, "Annual Review of Physical Chemistry, vol. 52: pp. 107-137, 2001. ( doi:10.1146/annurev.physchem.52.1.107)

[24] M. Scherge, J.A. Schaefer, "Microtribological investigations of stick/slip phenomena using a novel oscillatory friction and adhesion tester," Tribology Letters, vol. 4, no.1, pp. 37-42. 1998

[25] M. Jaschke, H.J. Butt, H.E. Gaub, S. Manne, "Surfactant aggregates at a metal surface," Langmuir, vol. 13, no. 6, pp. 1381-1384. 1997. ( doi: 10.1021/la9607767)

[26] J.H. Schon, Z. Bao, "Nanoscale organic transistors based on self-assembled monolayers," Applied Physics Letters, vol. 80, no. 5, pp. 847-849, 2002.

[27] N.K. Chaki, K. Vijayamohanan, "Self-assembled monolayers as a tunable platform for biosensor applications," Biosensors \& Bioelectronics, vol. 17, no.1-2, pp. 1-12, 2002.

[28] I. Doudevski, D.K. Schwartz, "Self-assembled monolayers in the context of epitaxial film growth," Applied Surface Science, vol. 175, pp. 17-26, 2001.

[29] N.K. Chaki, K. Vijayamohanan, "Self-assembled monolayers as a tunable platform for biosensor applications," Biosensors \& Bioelectronics, vol. 17, no. 1-2, pp. 1-12, 2002.

[30] G. Ceotto, E.F. de Souza, O. Teschke, "Ionic surfactant films imaged by atomic force microscopy," Journal of Molecular Catalysis a-Chemical, 167(1-2): p. 225-233, 2001

[31] F. Tiberg,, J. Brinck, L. Grant, "Adsorption and surface-induced self-assembly of surfactants at the solidaqueous interface," Current Opinion in Colloid \& Interface Science, vol. 4, no. 6, pp. 411-419, 2000. (https://doi.org/10.1016/S1359-0294(00)00016-9)

[32] Y.L. Chen, J.N. Israelachvili, "Effects of Ambient Conditions on Adsorbed Surfactant and Polymer Monolayers," Journal of Physical Chemistry, vol. 96, no. 19, pp. 7752-7760, 1992. (doi: 10.1021/j100198a048)

[33] S. Manne, J. P. Cleveland, H. E. Gaub, G. D. Stucky, P. K. Hansma, "Direct Visualization of Surfactant Hemimicelles by Force Microscopy of the Electrical Double-Layer," Langmuir, vol. 10, no. 12, pp. 4409-4413, 1994. (doi: 10.1021/la00024a003)

[34] Nishimura, S., P.J, Scales, S.R. Biggs, T.W. Healy, " AFM Studies of Amine Surfactant Hemimicelle Structures at the mica-water interface, " Colloids and Surfaces a-Physicochemical and Engineering Aspects, vol. 103, no. 3, pp. 289-298, 1995. https://doi.org/10.1016/0927-7757(95)03256-D

[35] Li, B.Y., M. Fujii, K. Fukada, T. Kato, T. Seimiya, "Time dependent anchoring of adsorbed cationic surfactant molecules at mice/solution interface, " Journal of Colloid and Interface Science, vol. 209, no. 1, pp. 25-30, 1999. (doi. 10.1006/jcis.1998.5869)

[36] N.K. Chaki, M. Aslam, J. Sharma, K. Vijayamohanan, "Applications of self-assembled monolayers in materials chemistry, " Proceedings of the Indian Academy of Sciences-Chemical Sciences, vol. 113, no. 5-6, pp. 659-670, 2001.

[37] J. Manojlovic, "Molecular mechanisms in boundary lubrication, SERBIATRIB '17, 15th International Conference on Tribology, Kragujevac, Serbia, pp. 469-474, 2017

[38] D. Brovelli, G. Hähner, L. Ruiz, R. Hofer, G. Kraus, A. Waldner, J. Schlösser, P. Oroszlan, M. Ehrat, N.D. Spencer, "Highly oriented, self-assembled alkanephosphate monolayers on tantalum (V) oxide surfaces," Langmuir, vol.15, no.13, pp. 4324-4327, 1999. doi: 10.1021/la981758n

[39] C. P.Whitby, P.J. Scales, F. G. Thomas, W.H. Nishimura, H. Tateyama, "The adsorption of dodecyltrimelhylammonium bromide on mica in aqueous solution studied by X-ray diffraction and atomic force microscopy", Journal of Colloid and Interface Science, vol. 235, no.2, pp. 350-357, 2001. https://doi.org/10.1006/jcis.2000.7359

[40] Y.L. Chen, J.N. Israelachvili, "Effects of Ambient Conditions on Adsorbed Surfactant and Polymer Monolayers", Journal of Physical Chemistry, 96(19): p. 7752-7760, 1992.

[41] Y.L. Chen, Helm C.A., J.N. Israelachvili, "Molecular Mechanisms Associated with Adhesion and Contact-Angle Hysteresis of Monolayer Surfaces", Journal of Physical Chemistry, vol. 95, no. 26, pp. 10736-10747, 1991.

[42] Y.I. Rabinovich, I.U. Vakarelski, S.C. Brown, P.K. Singh, B.M. Moudgil, "Mechanical and thermodynamic properties of surfactant aggregates at the solid-liquid interface, " Journal of Colloid and Interface Science, vol. 270, no.1, pp. 29-36, 2004. 